\title{
Two-Stage Fuzzy Fusion With Applications to Through-the-Wall Radar Imaging
}

\author{
Cher Hau Seng, Student Member, IEEE, Abdesselam Bouzerdoum, Senior Member, IEEE, \\ Moeness G. Amin, Fellow, IEEE, and Son Lam Phung, Member, IEEE
}

\begin{abstract}
A two-stage fuzzy image fusion approach, which combines multiple radar images of the same scene, is proposed to produce a more informative image. In this approach, two different image fusion methods are first applied. Then, a fuzzy logic fusion method is applied to the outputs of the first fusion stage. The performance of the proposed approach is evaluated on through-the-wall radar images obtained using different polarizations. Experimental results show that the proposed approach enhances image quality by producing outputs with high target intensity values and low clutter.
\end{abstract}

Index Terms-Fuzzy logic, image fusion, through-the-wall radar imaging (TWRI).

\section{INTRODUCTION}

$\mathbf{I}$ $\mathrm{N}$ REMOTE sensing applications, through-the-wall radar imaging (TWRI) systems are used to detect the presence of targets behind obstacles [1]. During the sensing process, the scene can be imaged with different polarizations from the same viewing angle. The scene may be also imaged from different viewing angles, using one or multiple systems. The acquired images are then fused to produce a more informative composite image of the scene [2].

Commonly used image fusion techniques in radar imaging include pixel-wise additive fusion [3], multiplicative fusion [4], wavelet transform (WT) fusion [5], principal component analysis (PCA) fusion [6], and fuzzy logic fusion [7]. There are also methods that use the maximum operator for fusion [8]. Compared with other methods, the fuzzy logic method adaptively fuses different parts of the image, depending on the relative presence of target and clutter. In addition, the fuzzy logic method produces output images with high target intensities and low clutter levels. However, the clutter levels produced by the fuzzy logic are not as low as those of the multiplicative fusion. In addition, the method proposed in [7] uses a fuzzy inference system (FIS) that maps from only two inputs to one output. In practice, the number of images acquired from different viewing angles and polarizations can exceed this limit. Although the FIS can be extended to map multiple

Manuscript received March 23, 2012 revised August 6, 2012; accepted August 30, 2012. Date of publication October 26, 2012; date of current version November 30, 2012. This work was supported in part by a grant from the Australian Research Council.

C. H. Seng, A. Bouzerdoum, and S. L. Phung are with the School of Electrical, Computer and Telecommunications Engineering, University of Wollongong, Wollongong, N.S.W. 2522, Australia (e-mail: aseng@uow.edu.au; a.bouzerdoum@uow.edu.au; phung@uow.edu.au).

M. G. Amin is with the Center for Advanced Communications, Villanova University, Villanova, PA 19085 USA (e-mail: moeness.amin@ villanova.edu).

Color versions of one or more of the figures in this paper are available online at http://ieeexplore.ieee.org.

Digital Object Identifier 10.1109/LGRS.2012.2218570 input images to one output, the complexity associated with formulating membership functions (MFs) and defining fuzzy rules significantly increases.

In this letter, we propose a two-stage fuzzy fusion (TSFF) approach that combines multiple images without the increased complexity. The images are generated using backprojection without applying any high-resolution technique [9]. The proposed approach is also used to enhance the fusion results of existing methods. In the first stage, the input images are combined using a pair of image fusion methods, such as additive and multiplicative, or wavelets and PCA. In the second stage, the fuzzy logic method is used to fuse the outputs of the first stage. Through this two-stage fusion, an enhanced composite image with high target intensities and lower clutter levels is produced.

The proposed TSFF approach is evaluated on polarimetric images collected at the Center for Advanced Communications, Villanova University. The performance of the proposed approach is compared with the additive, multiplicative, wavelets, and PCA fusion methods. The remainder of this letter is structured as follows. Section II reviews the existing image fusion methods that are commonly used in TWRI and synthetic aperture radar imaging, which are performed in the first fusion stage. Section III then presents the second-stage fuzzy fusion approach. Section IV analyzes the performance of the proposed approach and presents experimental results on real data. Section V concludes this letter.

\section{RADAR IMAGE FUSION}

Here, we review the four commonly used image fusion methods for radar imaging. The first two are the arithmetic fusion methods, namely, the additive and multiplicative fusion. The other two are the wavelets and the PCA, which are transformbased fusion methods. These four methods are applied during the first fusion stage.

\section{A. Arithmetic Fusion}

Arithmetic image fusion methods, such as additive and multiplicative, were proposed to improve TWRI. Additive fusion was first introduced for TWRI in [3] to correct for target displacements that are caused by unknown wall parameters. It was shown that the fusion of images obtained from different standoff positions reveals the exact location of the targets. The work in [4] later considered moving the antenna array around a building and combining the images through multiplicative fusion to improve detection and localization of indoor targets. The multiplicative fusion has been also applied for multichannel fusion [10] in TWRI applications. Both additive and multiplicative fusion methods were also used in [11] and 
[12] to enhance polarimetric radar by combining the outputs obtained from copolarization and cross-polarization imaging. Let $I_{j}(k, l)$ denote the $j$ th input image normalized to range $[0,1]$. The arithmetic fusion methods produce the additive image $I_{A}(k, l)$ and the multiplicative image $I_{M}(k, l)$ as follows:

$$
\begin{aligned}
I_{A}(k, l) & =\frac{1}{N} \sum_{j=1}^{N} I_{j}(k, l) \\
I_{M}(k, l) & =\prod_{j=1}^{N} I_{j}(k, l)
\end{aligned}
$$

where $N$ is the number of input images.

\section{B. WT Fusion}

The input images are first decomposed into subbands using the discrete WT (DWT). The subband coefficients are then fused together before applying the inverse DWT for image reconstruction [5]. Let $\mathrm{HH}_{j}, \mathrm{HL}_{j}, \mathrm{LH}_{j}$, and $\mathrm{LL}_{j}$ denote the subband coefficients of input image $j$. The coefficients of the high-frequency subbands, namely, HH, HL, and LH, are fused using the maximum operator, i.e.,

$$
\begin{aligned}
& \tilde{\mathrm{HH}}(k, l)=\max _{j}\left\{\left|\mathrm{HH}_{j}(k, l)\right|\right\} \\
& \tilde{\mathrm{HL}}(k, l)=\max _{j}\left\{\left|\mathrm{HL}_{j}(k, l)\right|\right\} \\
& \tilde{\mathrm{LH}}(k, l)=\max _{j}\left\{\left|\mathrm{LH}_{j}(k, l)\right|\right\} .
\end{aligned}
$$

The low-frequency subband coefficients, namely, $\operatorname{LL}_{j}(j=$ $1, \ldots, N)$, are fused together by a linear combination, i.e.,

$$
\tilde{\mathrm{LL}}(k, l)=\sum_{j=1}^{N} w_{j} \mathrm{LL}_{j}(k, l) .
$$

The weight $w_{j}$ is proportional to the mean $\ell_{j}$ of subband $\mathrm{LL}_{j}$, i.e.,

$$
w_{j}=\frac{\ell_{j}}{\sum_{j=1}^{N} \ell_{j}} .
$$

The final image $I_{W}$ is reconstructed from the fused subband coefficients $\tilde{\mathrm{HH}}, \tilde{\mathrm{HL}}, \tilde{\mathrm{LH}}$, and $\tilde{\mathrm{LL}}$ by taking the inverse DWT.

\section{PCA Fusion}

The PCA fusion method calculates the output image as a weighted sum of input images, where the weights are the elements of the dominant eigenvector [6]. The set of $N$ input images is organized into a matrix $\mathbf{X}$, where each row is a lexicographically ordered input image. Let vector $\boldsymbol{\mu}$ be the average of all the rows in $\mathbf{X}$, and let $\mathbf{e}$ be a column vector with all elements equal to 1 . The covariance matrix is given by

$$
\mathbf{C}=\frac{1}{M}\left(\mathbf{X}-\boldsymbol{\mu} \mathbf{e}^{T}\right)\left(\mathbf{X}-\boldsymbol{\mu} \mathbf{e}^{T}\right)^{T}
$$

where $M$ is the total number of pixels in an image. The fused image is given by

$$
I_{P}(k, l)=\sum_{j=1}^{N} v_{j} I_{j}(k, l)
$$

where $v_{j}$ is the $j$ th component of the dominant eigenvector, corresponding to the largest eigenvalue of $\mathbf{C}$.

\section{FUZZY FUSION APPROACH}

The proposed TSFF approach exploits the capabilities of the arithmetic or transform-based methods to produce an enhanced composite image. After fusing together the input images with two different methods, such as additive and multiplicative, or wavelets and PCA, the fuzzy fusion is applied to the two output images from the first stage. In the fuzzy fusion stage, the two images are normalized and then converted into membership values based on a set of predefined MFs, where the degree of membership of each input pixel to a fuzzy set is determined. Next, fusion operators are applied to the fuzzified images. The fusion results are then converted back into pixel values using defuzzification.

Radar images, in general, contain targets of interest, clutter/sidelobes, and noise. The clutter/sidelobe region overlaps in intensity with the target region on one side and with the noise region on the other. The target region could be also divided into strong and weak targets. The boundary between the target and noise regions is determined using entropy-based image segmentation. First, a probability mass function is constructed from the intensity histogram of the input images. Let $p_{i}$ be the probability of the $i$ th histogram bin, and let $P_{\tau}=\sum_{i=1}^{I_{\tau}} p_{i}$ be the cumulative probability, where the integer $I_{\tau}$ is the number of histogram bins containing intensity values smaller than or equal to the intensity threshold $\tau$. In other words, the cumulative probability $P_{\tau}$ represents the fraction of pixels whose intensity values are smaller than or equal to $\tau$. Suppose that image $I$ is to be divided into two regions $R_{1}$ and $R_{2}$ using $\tau$

$$
\begin{aligned}
& R_{1}(k, l)= \begin{cases}1, & \text { if } I(k, l) \leq \tau \\
0, & \text { otherwise }\end{cases} \\
& R_{2}(k, l)=1-R_{1}(k, l) .
\end{aligned}
$$

The entropies of $R_{1}$ and $R_{2}$, respectively, are given by

$$
\begin{aligned}
& H_{1}(\tau)=\ln P_{\tau}-\frac{1}{P_{\tau}} \sum_{i=1}^{I_{\tau}} p_{i} \ln p_{i} \\
& H_{2}(\tau)=\ln \left(1-P_{\tau}\right)-\frac{1}{\left(1-P_{\tau}\right)} \sum_{i=I_{\tau}+1}^{B} p_{i} \ln p_{i}
\end{aligned}
$$

where $B$ is the total number of histogram bins. The optimum threshold is found by maximizing the sum of the entropies of regions $R_{1}$ and $R_{2}$, i.e.,

$$
\tau^{*}=\arg \max _{\tau}\left\{H_{1}(\tau)+H_{2}(\tau)\right\} .
$$

Let $\tau_{1}$ be the optimum threshold obtained by concatenating all input images into a large composite image $I$. This threshold determines the boundary between the target and noise regions. To segregate the noise region from the clutter region, we use entropy segmentation again to partition region $R_{1}$ into two subregions: $R_{1 n}$ for noise and $R_{1 c}$ for clutter. The optimum threshold, which defines the boundary between $R_{1 n}$ and $R_{1 c}$, is hereafter denoted $\tau_{2}$. Given the two boundary points $\tau_{1}$ and $\tau_{2}$, the fuzzy MFs of three regions are formulated as follows:

$$
\begin{aligned}
& \mu_{1}(x)=\max \left\{\min \left\{-\left(x-\tau_{1}\right) /\left(\tau_{1}-\tau_{2}\right), 1\right\}, 0\right\} \\
& \mu_{3}(x)=\max \left\{\min \left\{\left(x-\tau_{1}\right) /\left(\tau_{1}-\tau_{2}\right), 1\right\}, 0\right\} \\
& \mu_{2}(x)=1-\left[\mu_{1}(x)+\mu_{3}(x)\right]
\end{aligned}
$$

where $x$ is the pixel value. 
In the fuzzification process, each input pixel is represented by its membership values $\mu_{1}, \mu_{2}$, and $\mu_{3}$. These membership values are then combined using fuzzy fusion rules. Let $\mu_{r, j}$ be the membership value associated with region $r$ of input image $j$, and let $\eta_{r}$ be the fused membership value. Instead of applying a global operator as in additive, multiplicative, wavelets, and PCA fusion, each pair of membership values $\left\{\mu_{r, 1}, \mu_{r, 2}\right\}$ is fused according to their associated regions. For instance, when one of the membership values is associated with the strong or weak target region, $\eta_{r}$ will be enhanced or maintained as a target. Similarly, when one of the membership values originates from the noise or clutter/sidelobe region, we suppress or maintain $\eta_{r}$ as noise. For the target and noise regions $(r=1,3)$, we employ the probabilistic OR operator to enhance the degree of membership of $\eta_{r}$ during fusion. While we could also use the same operator for the clutter/sidelobe region, the probabilistic OR will over-enhance the clutter or sidelobes. Thus, we chose the complement square root of a product operator to enhance the degree of membership of $\eta_{2}$. The fuzzy operators used are summarized as follows:

$$
\eta_{r}= \begin{cases}\mu_{r, 1}+\mu_{r, 2}-\mu_{r, 1} \mu_{r, 2}, & \text { if } r=1,3 \\ 1-\sqrt{\left(1-\mu_{r, 1}\right)\left(1-\mu_{r, 2}\right)}, & \text { if } r=2 .\end{cases}
$$

For each pixel, the fused degrees of membership, i.e., $\eta_{r}(r=$ $1, \ldots, 3)$, are defuzzified into pixel intensity values using the following mapping:

$$
I_{F}= \begin{cases}\tau_{1}+\left(I_{\max }-\tau_{1}\right) \eta_{3}, & \text { if } \eta_{3}>0 \\ \tau_{1} \eta_{2}, & \text { if } \eta_{3}=0 \text { and } \\ & \max \left\{\mu_{2,1}, \mu_{2,2}\right\} \geq 0.5 \\ \tau_{1}\left(1-\eta_{1}\right), & \text { otherwise }\end{cases}
$$

where $I_{F}$ is the fused intensity value, and $I_{\max }$ is the maximum gray-level value.

\section{ExPERIMEntal Methods And Results}

The proposed TSFF approach is evaluated on polarimetric TWR images collected at the Center for Advanced Communications, Villanova University. The performances of the proposed approach utilizing additive/multiplicative and wavelets/PCA are analyzed and compared with the additive, multiplicative, wavelets, and PCA image fusion methods. Before presenting the experimental results and analysis, the next section introduces the experimental procedure for data collection, followed by the performance measures employed to assess the quality of the fused images.

\section{A. Experimental Methods}

A scene containing isolated reflectors was constructed in the laboratory. Fig. 1 shows the eight targets placed at different down range, cross range, and elevations: 1) a sphere with a radar cross section (RCS) of $-11.4 \mathrm{dBm}^{2} ; 2$ ) three square-plate dihedrals, which are rotated by $22.5^{\circ}, 45^{\circ}$, and $90^{\circ}$, respectively, with an RCS of $11.3 \mathrm{dBm}^{2}$; 3) a 152.4-mm triangular-plate trihedral with an RCS of $-8.5 \mathrm{dBm}^{2}$; 4) two 76.2-mm triangularplate trihedrals with an RCS of $-20.6 \mathrm{dBm}^{2}$; and 5) a top hat, which is composed of a $127-\mathrm{mm}$-diameter cylinder and a 711.2-mm circular plate, with a maximum RCS of $4.87 \mathrm{dBm}^{2}$.

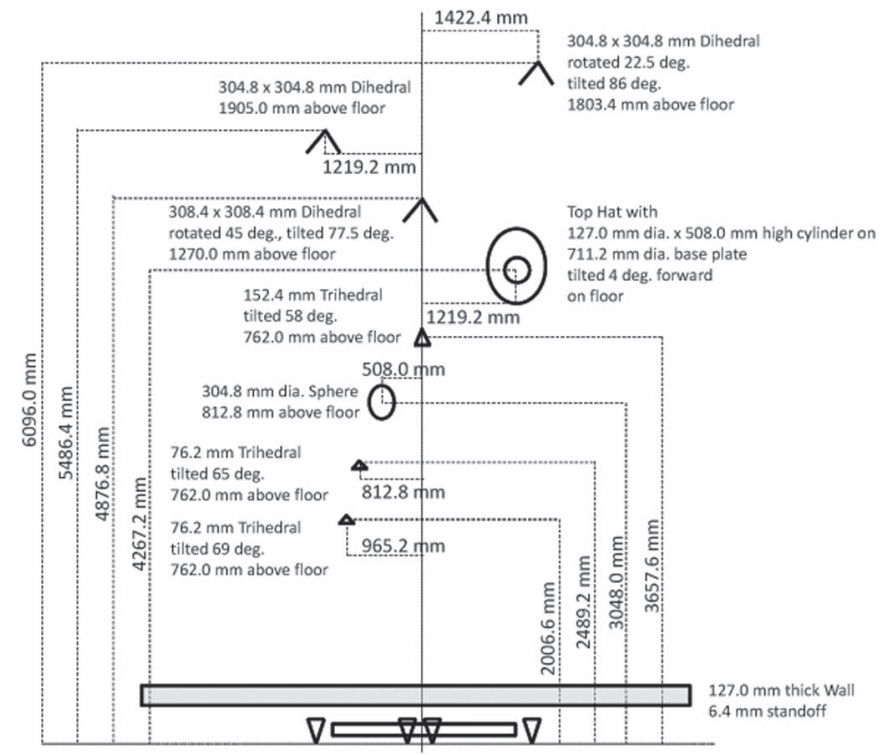

Fig. 1. Schematic of the scene (top view) with targets drawn to scale. Unless otherwise indicated, the heights shown are to the center of objects, and the tilt angles are $90^{\circ}$

Both copolarization ( $\mathrm{HH}$ and $\mathrm{VV})$ and cross-polarization ( $\mathrm{HV}$ and $\mathrm{VH}$ ) data sets were collected from the scene. For each polarization, the scene was imaged using a 57-element linear array, with an inter-element spacing of $22 \mathrm{~mm}$, utilizing a $1-\mathrm{GHz}$ bandwidth stepped-frequency waveform centered at $2.5 \mathrm{GHz}$, through a $127-\mathrm{mm}$-thick nonhomogeneous plywood and gypsum board wall. Fig. 2 shows the four images reconstructed using delay-and-sum beamforming. It can be observed that only two targets are present in the $\mathrm{HV}$ and $\mathrm{VH}$ images [see Fig. 2(b) and (c)]. The reason is that the rotated dihedrals produce a stronger cross-polarization return than the other targets in the scene.

\section{B. Performance Measures}

The performances of the image fusion methods are assessed in terms of computational time and image quality. The image quality is measured using the improvement factor of the targetto-clutter ratio (IF) and the target improvement factor (TIF). Let $\mathcal{P}_{r, q}$ denote the average power of region $r$ in image $I_{q}$, where $r$ is a target or clutter region, and $q$ is the input or output image. The average power $\mathcal{P}_{r, q}$ can be expressed as

$$
\mathcal{P}_{r, q}=\frac{1}{M_{r}} \sum_{(k, l) \in r} I_{q}^{2}(k, l)
$$

where $M_{r}$ is the number of pixels in region $r$. The IF is given by

$$
\mathrm{IF}=10 \log _{10}\left[\frac{\mathcal{P}_{\text {target,output }} / \mathcal{P}_{\text {clutter,output }}}{\mathcal{P}_{\text {target,input }} / \mathcal{P}_{\text {clutter,input }}}\right]
$$

and the TIF is defined as

$$
\mathrm{TIF}=10 \log _{10}\left[\frac{\mathcal{P}_{\text {target,output }}}{\mathcal{P}_{\text {target,input }}}\right] .
$$




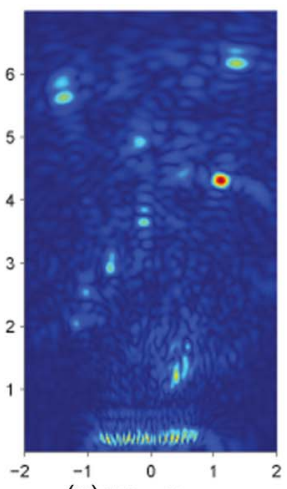

(a) $\mathrm{HH}$ Input Image

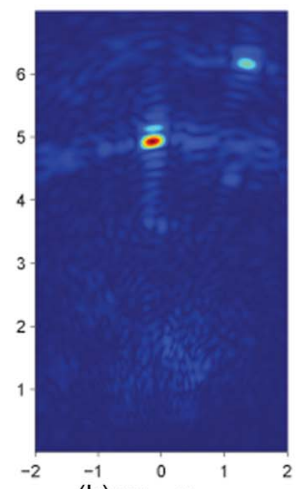

(b) HV Input Image

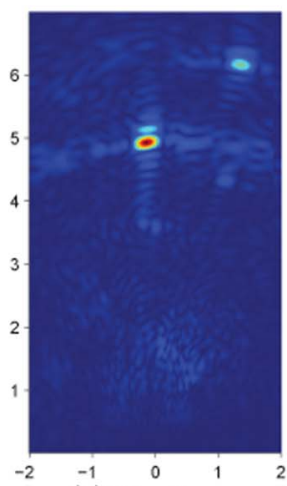

(c) VH Input Image

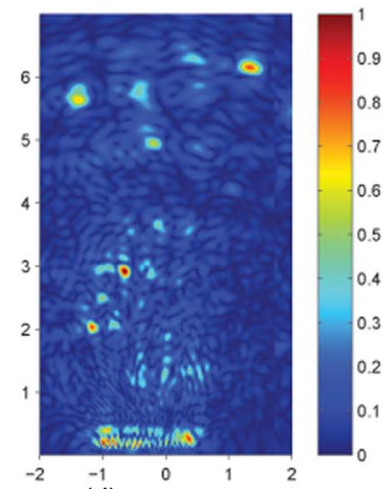

(d) W Input Image

Fig. 2. Input images of the same scene. The vertical and horizontal axes represent the down range and the cross range, with units in meters.

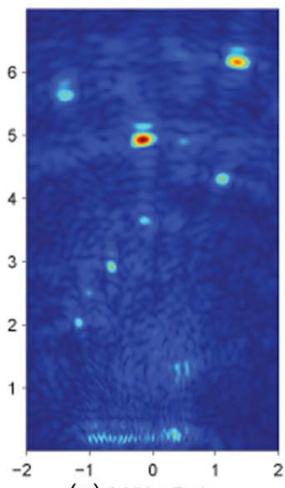

(a) Additive Fusion

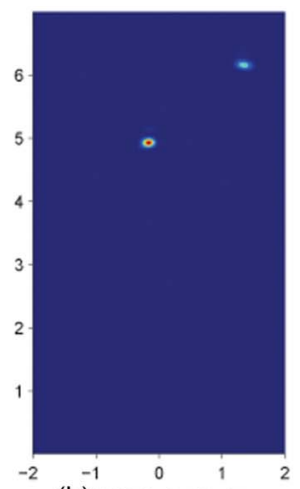

(b) Multiplicative Fusion

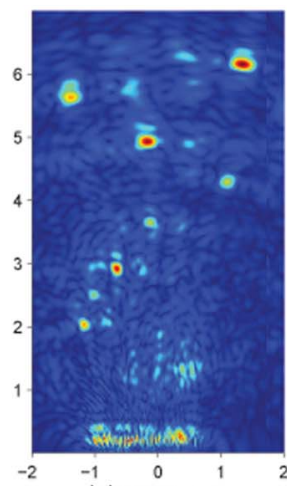

(c) DWT Fusion

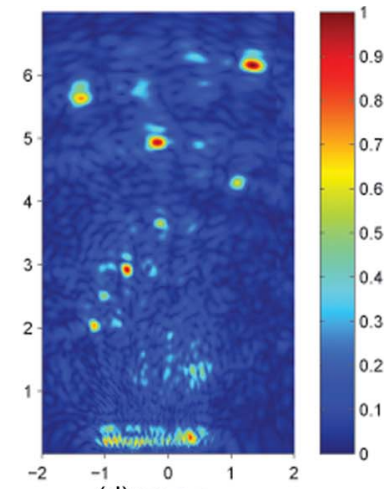

(d) PCA Fusion

Fig. 3. Output images produced by existing fusion methods.

TABLE I

Performance Measures of the Different Image Fusion Methods

\begin{tabular}{|l|r|r|r|}
\hline Method & $\begin{array}{c}\text { IF } \\
(\mathbf{d B})\end{array}$ & $\begin{array}{c}\text { TIF } \\
(\mathbf{d B})\end{array}$ & $\begin{array}{c}\text { Time } \\
(\mathbf{m s})\end{array}$ \\
\hline Additive Fusion & 0.00 & 0.00 & 7.2 \\
\hline Multiplicative Fusion & 15.31 & -9.62 & 3.2 \\
\hline WT Fusion & -1.35 & 2.03 & 493.3 \\
\hline PCA Fusion & -1.33 & 2.04 & 27.6 \\
\hline TSFF $\left(\mathrm{A} \& \mathrm{M}, \tau_{1}=75, \tau_{2}=50\right)$ & 26.76 & 3.38 & 13.7 \\
\hline TSFF $\left(\mathrm{W} \& \mathrm{P}, \tau_{1}=115, \tau_{2}=65\right)$ & 11.70 & 4.08 & 13.4 \\
\hline
\end{tabular}

The target region consists of pixels associated with the eight targets. All other pixels, except for the wall residue, are considered as clutter. In this letter, we have used the averaged image as the input, which is the same as the image obtained with additive fusion. This image serves as a reference against which the improvement in image quality is assessed.

\section{Results of First-Stage Fusion}

After image registration and normalization, four existing methods are applied to fuse multiple polarization images shown in Fig. 2. The results produced by the additive, multiplicative, wavelets, and PCA fusion methods are shown in Fig. 3, and Table I shows the IF, TIF, and computation time of the four methods. From Fig. 3, it can be observed that the additive fusion method retains the noise and clutter from the input images. Similarly, both wavelets and PCA also retain most of the noise and clutter. As a result, while the three methods enhance the targets, the clutter levels are also increased. This effect is indicated by the negative IFs of the wavelets and PCA (see Table I).
As for multiplicative fusion, only two targets are maintained in the output image. This is because the multiplicative fusion suppresses pixels that are not colocated in the input images, and there are only two targets that appear in the cross-polarization images (see Fig. 2). As a result, the multiplicative fusion has the highest IF but the lowest TIF among all four existing methods, as shown in Table I. As can be observed from Fig. 3 and Table I, the additive, wavelets, and PCA fusion methods tend to maintain both the targets and clutter, whereas the multiplicative fusion is effective at reducing clutter, but it also weakens the target intensity. Hence, the proposed two-stage approach is used to exploit the characteristics of the image fusion methods.

\section{Results of Second-Stage Fuzzy Fusion}

In the second stage, we apply fuzzy fusion to the output images of the first stage. To limit the complexity of fuzzy rules, we consider only two input images to the fuzzy fusion engine. In particular, we combine additive with multiplicative (A\&M) and wavelet fusion with PCA (W\&P). Applying entropy segmentation to the input images shown in Fig. 2, we obtain the boundary points $\tau_{1}=115$ and $\tau_{2}=50$. Note that these values are determined from the input images, independent of the fusion methods employed in the first stage. However, different fusion methods perform differently in terms of target enhancement and clutter reduction; therefore, the values of $\tau_{1}$ and $\tau_{2}$ should be chosen accordingly.

To investigate the effects of $\tau_{1}$ and $\tau_{2}$, we fix one parameter and vary the second. These two parameters have complementary roles: $\tau_{1}$ controls the target enhancement level, and 

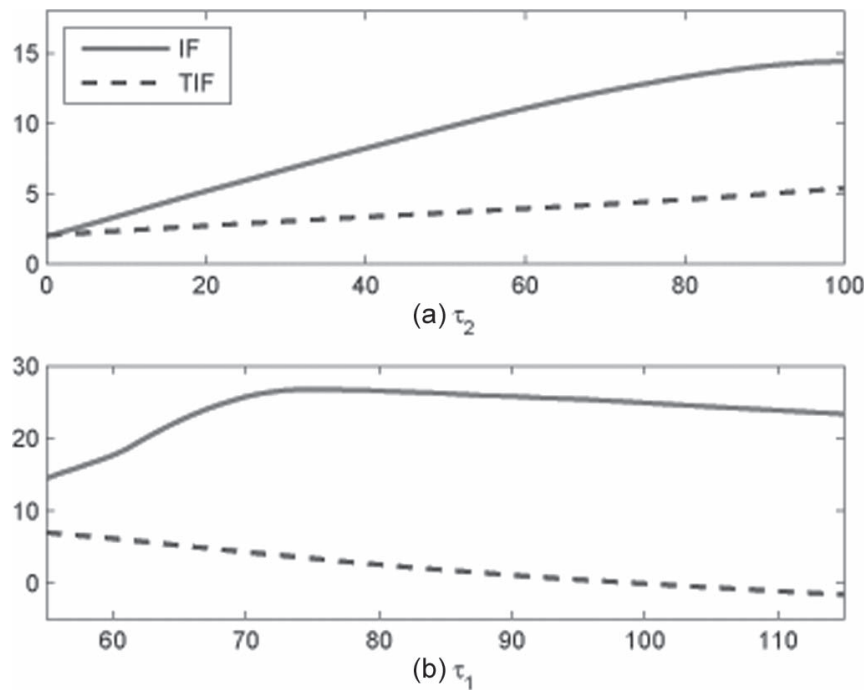

Fig. 4. Image enhancement measures of the proposed approach for different choices of (a) $\tau_{2}$ and (b) $\tau_{1}$.

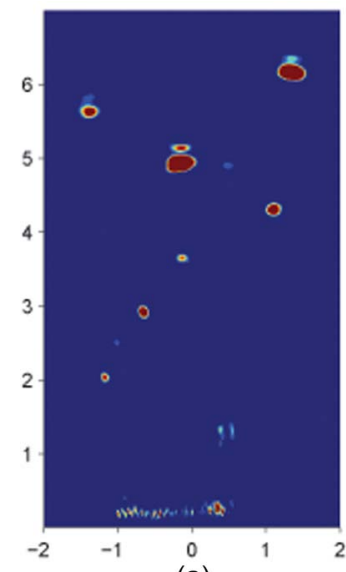

(a)

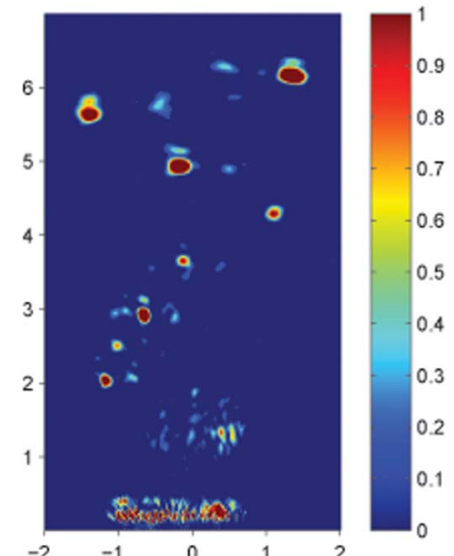

(b)
Fig. 5. Fused images of the scene, produced by the proposed TSFF approach utilizing (a) additive and multiplicative fusion and (b) WT and PCA fusion.

$\tau_{2}$ controls clutter reduction. We have seen in the previous section that both wavelets and PCA fusion maintain the targets at the expense of more clutter. In contrast, when combining multiplicative and additive fusion, the clutter is reduced, but weak targets are also suppressed. Therefore, to reduce clutter in W\&P fusion, we fix $\tau_{1}$ and vary $\tau_{2}$. Fig. 4(a) shows the IF and TIF of the W\&P TSFF when $\tau_{1}=115$ and $\tau_{2}$ is varied. Clearly, increasing $\tau_{2}$ results in more clutter reduction with little effect on the TIF. On the other hand, to enhance the weak targets suppressed by multiplicative fusion, we fix $\tau_{2}=50$ and vary $\tau_{1}$. Fig. 4(b) illustrates that the decrease in $\tau_{1}$ enhances the TIF of the A\&M fusion. However, there is also a decrease in IF. Hence, depending on the amount of clutter to suppress or target to enhance, different $\tau$ values can be used.

As an example, we fix $\tau_{2}=50$ and choose $\tau_{1}=75$ to enhance the weak targets in A\&M fusion, and we fix $\tau_{1}=115$ and choose $\tau_{2}=65$ to reduce the clutter in W\&P fusion. It can be observed from Fig. 5 that the proposed approach produces a balanced composite image that enhances target regions and reduces clutter. This is also evident from Table I, where both TSFF approaches produce output images with high IFs and maintain all the eight targets. In terms of target enhancements, the TSFF that combines the outputs of the wavelets and PCA produces an image with the highest TIF. This is because the proposed approach produces an output image with a high TIF when all the targets are maintained in the first fusion stage. Thus, similar results with a high TIF will be produced when using the two-stage approach to fuse additive and wavelets, or additive and PCA. Conversely, an output image with a lower TIF and a high IF will be produced when one of the input images has low clutter levels.

Table I shows the time taken for each fusion method, executed in MATLAB on a 3-GHz Core i7 CPU computer with 4-GB RAM. Note that the time shown for TSFF is only for the second-stage fusion without the entropy segmentation, which takes approximately $10 \mathrm{~ms}$. However, entropy segmentation is applied only once to determine the appropriate values of $\tau_{1}$ and $\tau_{2}$; subsequent fusion operations can use the same threshold values without recourse to entropy segmentation.

\section{CONCLUSION}

This letter has presented an effective TSFF approach to address the limitations of the standard fuzzy logic method and to enhance the results of existing image fusion methods. The proposed approach was applied to urban sensing, specifically to imaging of targets behind walls. Experimental results on real polarimetric data demonstrated the effectiveness of the proposed approach for pixel-level image fusion. Compared with the existing methods, the TSFF produces output images with high target intensities and low clutter levels, thus improving image quality and contrast.

\section{REFERENCES}

[1] M. Amin, Through the Wall Radar Imaging. Boca Raton, FL: CRC Press, 2010.

[2] L. Fonseca, L. Namikawa, and E. Castejon, "Digital image processing in remote sensing," in Proc. Tutorials 22th Braz. Symp. Comput. Graphics Image Process., 2009, pp. 59-71.

[3] G. Wang and M. Amin, "Imaging through unknown walls using different standoff distances," IEEE Trans. Signal Process., vol. 54, no. 10, pp. 4015-4025, Oct. 2006.

[4] F. Ahmad and M. Amin, "Multi-location wideband synthetic aperture imaging for urban sensing applications," J. Franklin Inst., vol. 345, no. 6 , pp. 618-639, Sep. 2008.

[5] S. Hong, W. Moon, H. Paik, and G. Choi, "Data fusion of multiple polarimetric SAR images using discrete wavelet transform (DWT)," in Proc. IEEE Int. Geosci. Remote Sens. Symp., 2002, pp. 3323-3325.

[6] A. Farina, F. Morabito, S. Serpico, and G. Simone, "Fusion of radar images: State of the art and perspective," in Proc. CIE Int. Conf. Radar, 2001, pp. 9-15.

[7] C. Seng, A. Bouzerdoum, F. Tivive, and M. Amin, "Fuzzy logic-based image fusion for multi-view through-the-wall radar," in Proc. Int. Conf. Digital Image Comput. Tech. Appl., 2010, pp. 423-428.

[8] R. Solimene, F. Soldovieri, and G. Prisco, "A multiarray tomographic approach for through-wall imaging," IEEE Trans. Geosci. Remote Sens., vol. 46, no. 4, pp. 1192-1199, Apr. 2008.

[9] Y. Yoon and M. G. Amin, "High-resolution through-the-wall radar imaging using beamspace MUSIC," IEEE Trans. Antennas Propag., vol. 56, no. 6, pp. 1763-1774, Jun. 2008.

[10] Y. Jia, L. Kong, X. Yang, and K. Wang, "Multi-channel through-wallradar imaging based on image fusion," in Proc. IEEE Radar Conf., 2011, pp. 103-105.

[11] F. Ahmad and M. Amin, "Through-the-wall polarimetric imaging," in Proc. SPIE, 2008, vol. 6970, pp. $69700 \mathrm{~N}-1-69700 \mathrm{~N}-10$.

[12] T. Dogaru and C. Le, "SAR images of rooms and buildings based on FDTD computer models," IEEE Trans. Geosci. Remote Sens., vol. 47, no. 5, pp. 1388-1401, May 2009. 